\title{
Novel OSNR Monitoring Technique in Dense WDM Systems using Inherently Generated CW Monitoring Channels
}

\author{
Petersen, Martin Nordal
}

Published in:

Conference on Optical Fiber Communication and the National Fiber Optic Engineers Conference, 2007. OFC/NFOEC 2007.

Link to article, DOI:

10.1109/OFC.2007.4348599

Publication date:

2007

Document Version

Publisher's PDF, also known as Version of record

Link back to DTU Orbit

Citation $(A P A)$ :

Petersen, M. N. (2007). Novel OSNR Monitoring Technique in Dense WDM Systems using Inherently Generated CW Monitoring Channels. In Conference on Optical Fiber Communication and the National Fiber Optic Engineers Conference, 2007. OFC/NFOEC 2007. (pp. OMM4). IEEE. https://doi.org/10.1109/OFC.2007.4348599

\section{General rights}

Copyright and moral rights for the publications made accessible in the public portal are retained by the authors and/or other copyright owners and it is a condition of accessing publications that users recognise and abide by the legal requirements associated with these rights.

- Users may download and print one copy of any publication from the public portal for the purpose of private study or research.

- You may not further distribute the material or use it for any profit-making activity or commercial gain

- You may freely distribute the URL identifying the publication in the public portal 


\title{
Novel OSNR Monitoring Technique in Dense WDM Systems using Inherently Generated CW Monitoring Channels
}

\author{
Martin Nordal Petersen and Torger Tokle \\ $C O M \cdot D T U$, Department of Communications, Optics, and Materials, Technical University of Denmark, \\ DK-2800 Kgs. Lyngby, Denmark.E-mail: mnp@com.dtu.dk, Telephone: +45 45256381.
}

\begin{abstract}
We present a simple, yet effective OSNR monitoring technique based on an inherent effect in the optical modulator. Highly accurate OSNR monitoring is demonstrated in a $40 \mathrm{~Gb} / \mathrm{s}$ dense WDM system with $50 \mathrm{GHz}$ channel spacing.

(C)2007 Optical Society of America

OCIS codes: 060.2330 Fiber optics communications; 070.4790 Optical spectrum analysis.
\end{abstract}

\section{Introduction}

The desire for higher capacity in optical communication systems has lead to increased per channel bit-rates as well as decreased channel spacing to enhance the spectral efficiency. At the same time, the realization of all-optical networks is moving closer with the introduction of optical switches and optical add-drop multiplexers, making accurate signal quality monitoring very difficult.

Traditionally, signal quality monitoring has been carried out in the electrical layer i.e. in nodes where the signal was optically regenerated. In networks without or with few optical-electrical-optical repeaters, methods for monitoring the quality of the optical signal directly are required [1]. One of the essential signal parameters to monitor is the optical-signal-to-noise-ratio (OSNR) that will provide important information about the condition of the optical signal. Traditionally, the OSNR can be measured using the so-called linear interpolation method where it is assumed that the noise level next to the channel is the same as the in-band noise level. However, this method becomes insufficient in dense WDM systems where the channel side-bands are effectively hiding the noise level [2]. Methods have been developed to overcome this problem, and in [3-5] it have been reported how the accuracy of OSNR monitoring can be improved under such circumstances using either polarization extinction [3], polarization nulling [4] or digital signal processing [5].

This paper presents a novel OSNR monitoring that utilizes inherently generated continuous wave (CW) monitoring tones, thus making it very simple but effective. By misaligning the polarization to the Mach-Zehnder (MZ) modulator, two orthogonal signals are generated; one with data modulation and one with a CW tone at the same wavelength. This CW tone can in term be used effective to monitor the OSNR very accurately in a dense spaced WDM network. We experimentally demonstrate this new method in a $40 \mathrm{~Gb} / \mathrm{s} 15$ channel dense WDM system with $50 \mathrm{GHz}$ channel spacing. With the new method we are able to measure OSNR above $30 \mathrm{~dB}$, whereas the traditional method is limited to approximately $10 \mathrm{~dB}$.

\section{Concept and principle}

The concept of the OSNR monitoring method is illustrated in Figure 1. By adjusting the polarization of the light incident to the MZ modulator only a part of the light is modulated as the modulator can only modulate light that is polarized in one certain linear state. The remaining light will go through the modulator without being modulated.

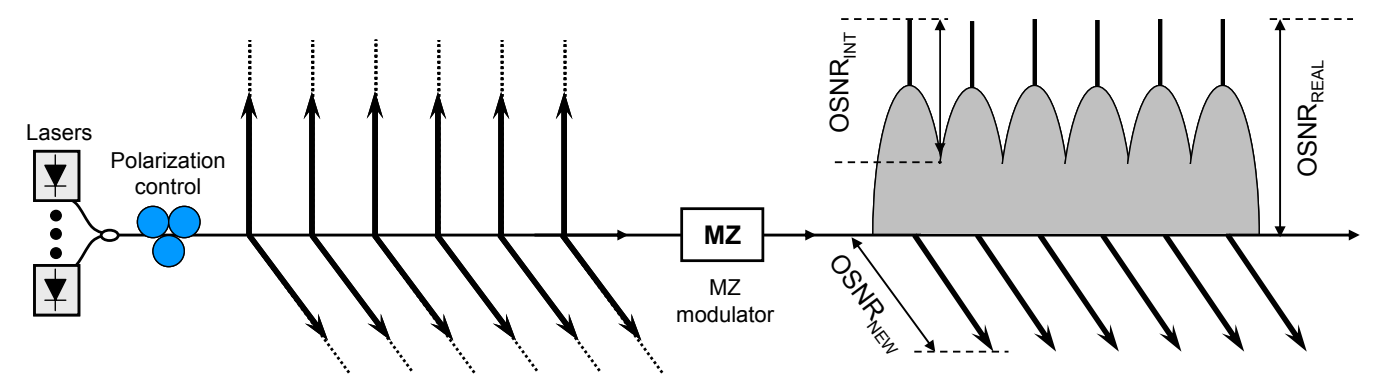

Figure 1. The figure illustrates the simple concept of the OSNR monitoring method using the inherently generated CW tones. By aligning the polarization if the incoming signals the modulator will only modulate part of the signal, i.e. one state of polarization. The remaining part will go though the modulator as a CW signal that in turn can be used for accurately monitoring OSNR in dense WDM systems. 


\section{OMM4.pdf}

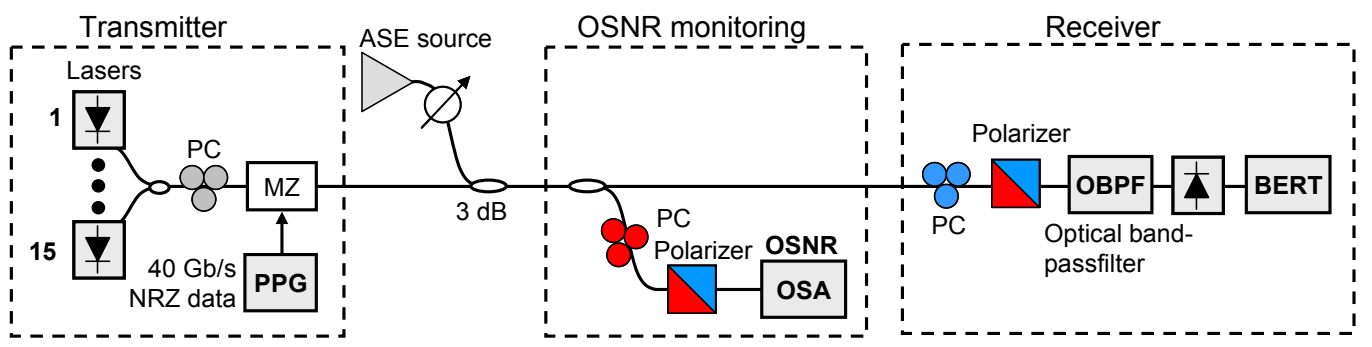

Figure 2. Setup used to demonstrate OSNR monitoring in a 15 channel, $50 \mathrm{GHz}$ channel spacing, $40 \mathrm{~Gb} / \mathrm{s} \mathrm{NRZ} \mathrm{system.}$

This can be seen from Figure 1 where all the data modulated channels are oriented along one polarization and only $\mathrm{CW}$ is present in the orthogonal polarization state. The figure also illustrates how using the traditional interpolation method results in an incorrect estimated $\mathrm{OSNR}_{\mathrm{INT}}$ as the side-bands of the neighboring channels overlap and thus hides the true noise level. Using the $\mathrm{CW}$ for OSNR evaluation instead achieves a very accurate $\mathrm{OSNR}_{\mathrm{NEW}}$ such that $\mathrm{OSNR}_{\mathrm{NEW}}$ ideally would equal the actual OSNR (OSNR $\left.\mathrm{REAL}\right)$. Also note that the concept is made possible as noise typically stays un-polarized and therefore will be equally present in both polarization states. In practice OSNR $\mathrm{NEW}_{\mathrm{NE}}$ found by first isolating the $\mathrm{CW}$ monitoring channels using a polarizer and then looking at the optical power spectrum of the signal. Also, in the receiver, a polarizer is used to remove the monitoring signal before detection. As polarizer's have a finite rejection of the orthogonal component of 40-50dB, typically which means that some modulated data will be visible when observing the $\mathrm{CW}$ spectrum. This, however, is not a problem for the method as will become evident below.

Figure 2 shows the experimental realization of the OSNR monitoring method. An array of 15 lasers are fed to the MZ modulator with an input state of polarization such that a signal consisting of both $40 \mathrm{~Gb} / \mathrm{s}$ modulated NRZ data and CW signals are produced. The OSNR of the transmitted signal can be adjusted by adding amplified spontaneous emission (ASE) noise from an optical noise source. Furthermore, using a gain flatting filter, the noise spectrum could be shaped such that different channels have a different OSNR. At the receiver end the data signals are separated from the monitoring $\mathrm{CW}$ signals using a polarizer. The data signals are detected in a photo diode whereas the monitoring $\mathrm{CW}$ channels are analyzed using an optical spectrum analyzer (OSA). It is here vital that the polarizer's are correctly adjusted such that the crosstalk between the two polarization states is minimized.

\section{Results}

To demonstrate the effectiveness of the method a number of OSNR monitoring experiments were carried out using the 15 channel WDM system setup shown in Figure 2. As noted above, the channel data is $40 \mathrm{~Gb} / \mathrm{s} \mathrm{NRZ}$ and the channel spacing is $50 \mathrm{GHz}$.

Figure $3 \mathrm{a}$ shows the relation between the Q-factor and the real OSNR, which was measured using the center channel (Ch\# 8) while shutting off two neighboring channels on each side. As the noise level was uniform in this experiment the interpolation method produced the real OSNR.

a)

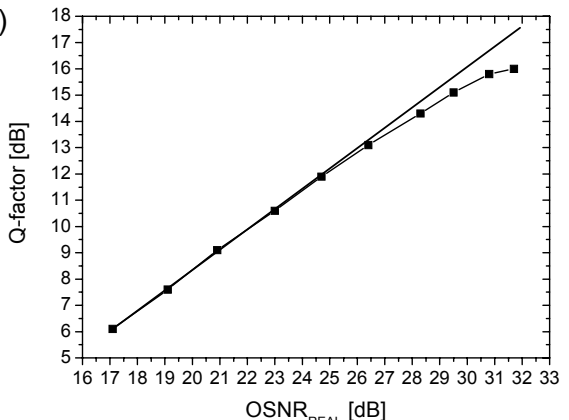

b)

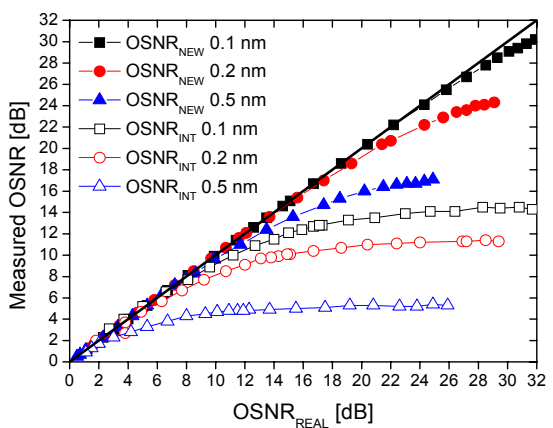

Figure 3. a) Q-factor versus OSNR for a $40 \mathrm{~Gb} / \mathrm{s} \mathrm{NRZ} \mathrm{signal.} \mathrm{OSNR} \mathrm{REAL}_{\text {was }}$ measured at resolution bandwidth $0.1 \mathrm{~nm}$. b) The OSNR for both the traditional interpolation method (OSNR $\mathrm{INT}_{\mathrm{IN}}$ ) and the new method $\left(\mathrm{OSNR}_{\mathrm{NEW}}\right)$ was measured versus the true OSNR. Notice how the OSNR $\mathrm{OSEW}$ strongly improves the OSNR evaluation accuracy for all resolution bandwidths: $0.1 \mathrm{~nm}, 0.2 \mathrm{~nm}$ and $0.5 \mathrm{~nm}$. 


\section{OMM4.pdf}

a)

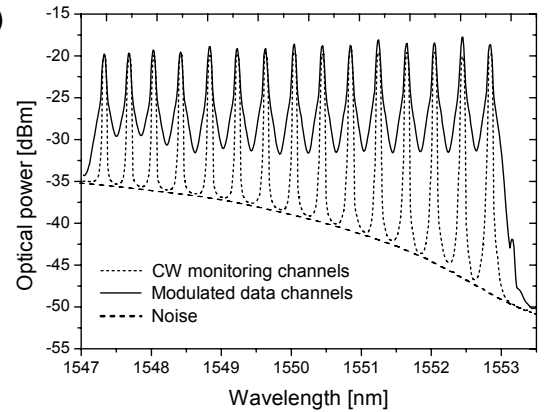

b)

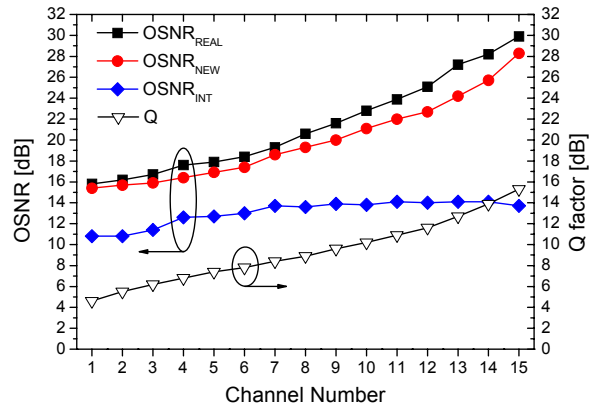

Figure 4. a) The figure shows a case of noise level tilt and how the method is still effective. The resolution bandwidth is 0.1 nm. b) Measurements showing how OSNR and Q-factor changes with channel number. The proposed method produces results that closely resemble the actual OSNR as well as providing information on the Q-factor through the linear dependence between OSNR $\mathrm{NEw}_{\text {and }}$ the Q-factor.

As expected, there is a linear relationship between the Q-factor and the OSNR of the signal. The deviation from the straight line seen at high OSNR is due to difficult optimization at very high Q-factors. The correlation between the Q-factor and OSNR underlines the importance of OSNR monitoring. Figure 3b shows the efficiency of the method as the interpolation method $\left(\mathrm{OSNR}_{\mathrm{INT}}\right)$ and the proposed method $\left(\mathrm{OSNR}_{\mathrm{NEW}}\right)$ has been plotted versus the true $\left(\mathrm{OSNR}_{\mathrm{REAL}}\right)$ for a resolution bandwidth of $0.1 \mathrm{~nm}, 0.2 \mathrm{~nm}$ and $0.5 \mathrm{~nm}$. The straight line with an inclination of 1 corresponds to an ideal OSNR measurement and it is seen that the proposed method is accurate for OSNR levels up to $30 \mathrm{~dB}$, whereas the interpolation method is only accurate up to around $10 \mathrm{~dB}$.

To further demonstrate the effectiveness of the proposed OSNR monitoring technique, an error level was created that had a spectral tilt as shown in Figure 4a. The bottom dashed curve represents the noise level in the system which was created by shaping the ASE noise source using a gain flattening filter. This situation simulates a realistic situation that can arise from for example EDFAs having uneven gain profiles. Figure 4a also shows the modulated data channels as well as the $\mathrm{CW}$ channels used for monitoring. Even at a resolution bandwidth of $0.1 \mathrm{~nm}$ a traditional OSNR evaluation is impossible due the spectral side-band overlap between the channels. One the other hand, using the CW monitoring channels, it is clearly seen how the noise level becomes visible and an accurate OSNR evaluation can be made. Figure $4 \mathrm{~b}$ shows the actual OSNR measurements performed on the situation illustrated in Figure 4a. The lower channels experiencing more noise than the higher channels are clearly affected as can be observer from the Q-factor measurements. The OSNR $\mathrm{INT}_{\mathrm{T}}$ curve corresponding to OSNR evaluations based on the interpolation method provides no useful information whereas the proposed method $\left(\mathrm{OSNR}_{\mathrm{NEW}}\right)$ achieves OSNR values equal to the actual OSNR $\left(\mathrm{OSNR}_{\mathrm{REAL}}\right)$. Also notice the desired linear correlation between the Qfactor and $\mathrm{OSNR}_{\mathrm{NEW}}$.

In terms of stability issues, the method faces similar problems as other OSNR monitoring techniques relating on polarization effects, namely influence from second order polarization mode dispersion (PMD) and wavelength dependant birefringence. Although not yet investigated, it is our belief that the majority of systems would not face these issues to an extent influence the results significantly.

\section{Conclusion}

A novel OSNR monitoring technique has been presented. The method uses CW monitoring signals inherently generated in the optical modulator by a simple adjustment of the incident state of polarization. Accurate OSNR evaluations up to $30 \mathrm{~dB}$ were demonstrated using a 15 channel $40 \mathrm{~Gb} / \mathrm{s}$ WDM signal with dense channel spacing. The method also proved accurate for the case of a tilted noise level where the traditional interpolation method could not predict the OSNR.

\section{References}

[1] D. C. Kilper, "Optical Performance Monitoring”, Jour. of Lightwave Tech., Vol. 22(1), pp. 294-304, 2004.

[2] M. Sköld et al., "PMD-insensitive DOP-based OSNR monitoring by spectral SOP measurements", OFC 2005, paper OThH3, 2005.

[3] M. Rasztovits-Wiech et al., "Optical signal-to-noise ratio measurement in WDM networks using polarization extinction," ECOC 98, pp. 549-550, 1998

[4] J. H. Lee et al., "OSNR Monitoring Technique Using Polarization-Nulling Method”, IEEE Phot. Tech. Lett., Vol. 13(1),pp. 88-90 2001.

[5] S. K. Shin, et al. "A novel optical signal-to-noise ratio monitoring technique for WDM networks", OFC 2000, pp. 182-184 paper WK6-1, 2000 